Dermatology 2012;224:209-211

DOI: $\underline{10.1159 / 000336775}$

\section{Labial Melanotic Macule: A Potential Pitfall on Reflectance Confocal Microscopy}

\author{
N. Erfan a , V. Hofman ${ }^{\text {b }}$, F. Desruelles ${ }^{\text {a }}$, T. Passeron a , J.P. Ortonne ${ }^{\text {a }}$, \\ J.P. Lacour ${ }^{\text {a }, ~ P . ~ B a h a d o r a n ~}{ }^{\mathrm{a}, \mathrm{c}}$ \\ Departments of a Dermatology, b Pathology and CINSERM U895 \\ Team, 9 Pasteur Hospital, University of Sophia-Antipolis, Nice, \\ France
}

\section{Key Words}

Labial melanotic macule - Reflectance confocal microscopy • Melanoma

\section{Introduction}

Labial melanotic macules (LMM) are benign pigmented lesions that can be difficult to differentiate from melanoma on the mucous membrane and the mucocutaneous junction $[1,2]$. LMM are secondary to a variable increase in melanin in the basal layer leading to hyperpigmentation of the basal keratinocytes along with an increase in the number of melanophages, and a normal or slightly increased number of melanocytes [2].

Reflectance confocal microscopy (RCM) is a novel non-invasive imaging technique that has gained substantial recognition because of its diagnostic uses in many skin pathologies.

Here we report the RCM findings in 4 cases of LMM and discuss the significance of these findings with regard to RCM criteria of other pigmented lesions.

\section{Case Reports}

Case 1. A 55-year-old woman presented with a history of a pigmented macule of the lower lip. The lesion appeared insidiously over a period of 6 months at the left lateral aspect of the lower lip. Clinical examination showed a brown-grey macule, measuring $5 \mathrm{~mm}$ (fig. 1a). Dermoscopy showed an ill-defined heterogeneously pigmented lesion (fig. 1b). RCM showed a normal epidermis with a honeycomb pattern and absence of atypical cells, and at the dermo-epidermal junction (DEJ) bright refractile cells surrounding the dermal papillae (fig. 1c). Notably, a significant proportion of these cells were melanocytes of variable size and shape and markedly branching dendrites. The lesion was biopsied, and histopathological evaluation showed a lentiginous background with hyperpigmented keratinocytes (fig. 1d). There was evidence of dermal elastosis and absence of cytonuclear atypia with regard to melano- cytes. Immunostaining with melan-A antibody confirmed a moderate augmentation in the number of basal melanocytes with branching dendrites extending into the suprabasal layers of the epidermis, sometimes resembling the spreading branches of a tree (fig. 1e).

Case 2. A 60-year-old man presented with a long-standing history of a pigmented macule of the lower lip. Clinical examination showed a brown homogeneous macule measuring $3 \mathrm{~mm}$ (fig. 2a). Dermoscopy showed a network pattern (fig. 2b). RCM showed a normal epidermis and numerous atypical dendritic cells at the DEJ (fig. 2c). Taking into consideration the benign clinical and dermoscopic presentations of the lesion, no biopsy was performed.

Case 3. A 26-year-old woman presented with a pigmented macule of the lower lip that had appeared 2 years before. The lesion was homogeneous, brown/black, measuring $4 \times 3 \mathrm{~mm}$ (fig. 3a). Dermoscopy showed a benign so-called hyphal pattern (fig. 3b) [1]. RCM showed a normal epidermis and dendritic cells at the DEJ (fig. 3c). The lesion remained unchanged during consecutive digital monitoring.

Case 4. A 37-year-old woman was referred for dermatological surveillance of her naevi. The patient was found to have 2 pigmented macules of the lower lip that had appeared 1 year prior to presentation (fig. 4a). Clinical examination and dermoscopy (fig. 4b) were not suspicious. RCM showed some dendritic cells at the DEJ (fig. 4c). The lesion remained unchanged during consecutive digital monitoring.

\section{Comments}

LMM are commonly seen in daily practice and may be difficult to differentiate from mucosal malignant melanoma. RCM, a novel non-invasive imaging technique, is now widely recognized as a reliable tool to discriminate between benign and malignant melanocytic proliferations of the skin.

Noteworthy, in our 4 cases of LMM, the more conspicuous RCM sign was the presence of abundant and highly dendritic cells at the DEJ which were confirmed to be normal melanocytes on histology. These findings are in accordance with previous histopathological studies of LMM which portrayed an increase in dendritic melanocytes in these lesions, confined to the basal layer, and without nest formation [2-4].

In actinic lentigines, the most common type of benign pigmented macules of the skin, dendritic cells, are not detectable by RCM [5], possibly because in these lesions the majority of melanocytes present in the basal layer of the epidermis are suspended in the dermis and are called 'pendulum melanocytes' [6]. On the other hand, the presence of dendritic melanocytes on RCM is considered to be an important diagnosis criterion of lentigo maligna [5].

\section{KARGER}

(๑) 2012 S. Karger AG, Basel

Fax +41613061234

E-Mail karger@karger.ch

www.karger.com
Accessible online at:

www.karger.com/drm
Philippe Bahadoran, $\mathrm{MD}, \mathrm{PhD}$

CHU Nice, Hôpital Archet 2

151, route Saint-Antoine-Ginestière

FR-06202 Nice (France)

Tel. +33 492036 223, E-Mail bahadoran.p @ chu-nice.fr 

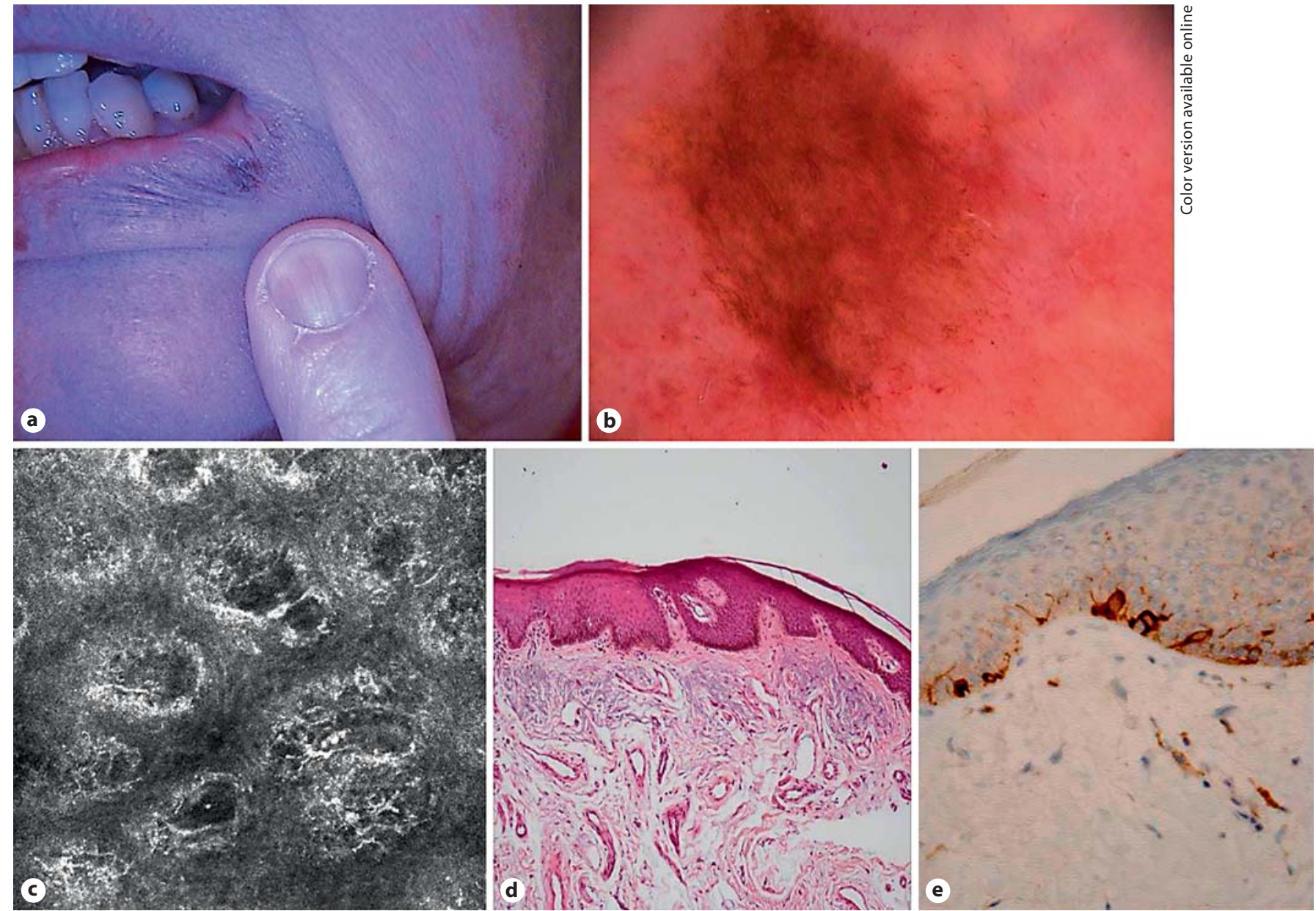

Fig. 1. a Clinical image of the patient's lower lip showing a grey-brown heterogeneous macule. b Dermoscopic image demonstrating non-specific amorphous pigmentation. c RCM image showing increased dermal papillae surrounded by large, atypical dendritic cells. $\mathbf{d}$ Histopathological image showing a lentiginous background with multiple hyperpigmented keratinocytes. $\times 100$. e Melan-A immunostaining showing an increase in highly dendritic melanocytes at the basal layer. $\times 400$.
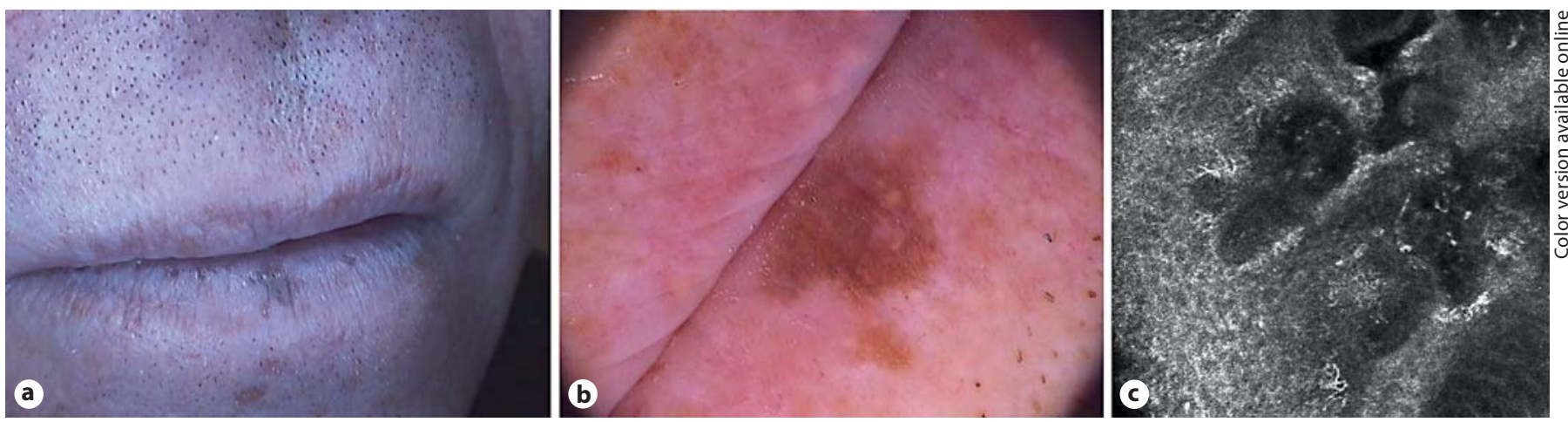

Fig. 2. a Clinical image of the patient's lower lip showing a small, discrete, homogeneously pigmented macule. b Dermoscopic image of the lesion showing a brown uniformly pigmented macule with a reticular pattern. c RCM image showing multiple atypical dendritic cells around the dermal papilla. 

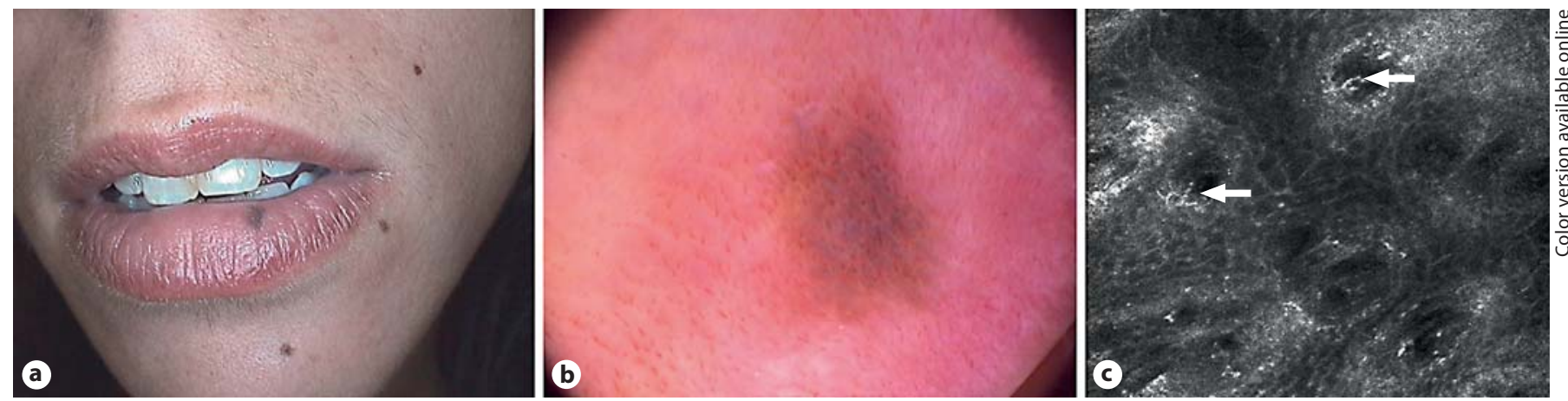

Fig. 3. a Clinical image of a pigmented macule of the lower lip showing a homogeneous, brown/black aspect and measuring $4 \times 3 \mathrm{~mm}$. b Dermoscopy showing a benign 'hyphal' pattern. c RCM showing a normal epidermis and dendritic cells at the DEJ (arrows).
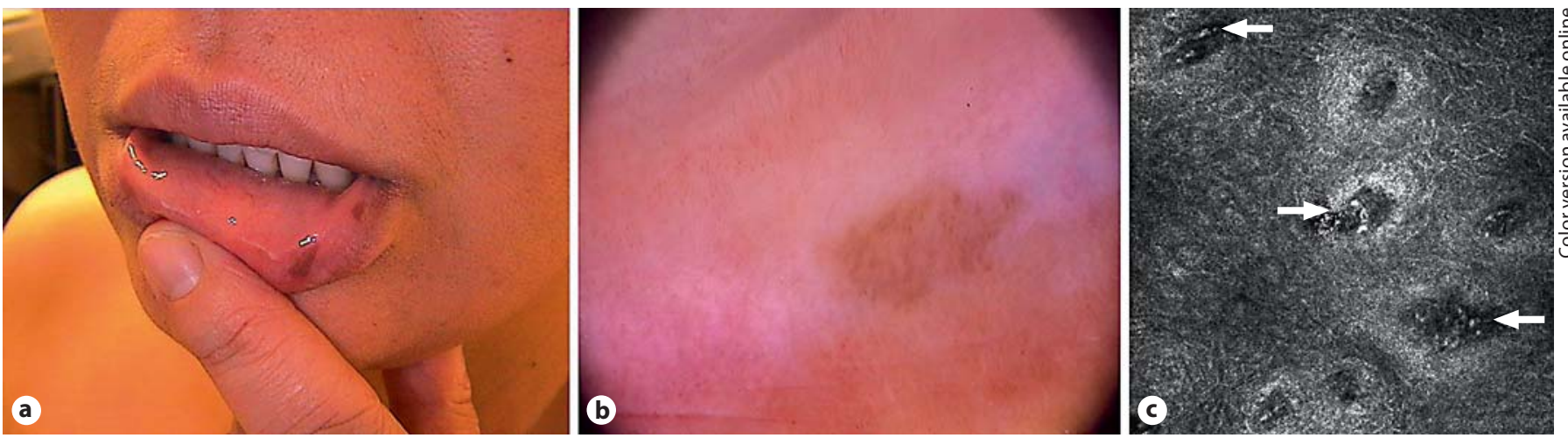

Fig. 4. a Clinical image showing two homogeneously pigmented macules of the lower lip. b Dermoscopy showing slightly pigmented macules with a reticular pattern. c RCM showing some dendritic cells at the DEJ (arrows).

In conclusion, LMM should not be confounded for a malignant lesion due to the presence on RCM of atypical dendritic cells within the epidermis. RCM criteria for lentigo maligna [5], when applied to such lesions, could lead to false positivity if these differences between skin and mucosa are overlooked. Additional studies are needed to allow the definition of specific RCM criteria for mucosal pigmented lesions.

\section{Disclosure Statement}

The authors have no conflict of interest to disclose.

\section{References}

1 Lin J, Koga H, Takata M, et al: Dermoscopy of pigmented lesions on mucocutaneous junction and mucous membrane. Br J Dermatol 2009; 161:1255-1261.

2 Ho KK, Dervan P, et al: Labial melanotic macule: a clinical histopathologic and ultrastructural study. J Am Acad Dermatol 1993;28:33-39.

3 Sexton FM, Maize JC: Melanotic macules and melanoacanthomas of the lip. A comparative study with census of the basal melanocyte population. Am J Dermatopathol 1987;9:438-444.

4 Moore RT, Chae KA, Rhodes AR: Laugier and Hunziker pigmentation: a lentiginous proliferation of melanocytes. J Am Acad Dermatol 2004; 50(suppl):S70-S74.

5 Noblesse E, Nizard C, Cario-André M, et al: Skin ultrastructure in senile lentigo. Skin Pharmacol Physiol 2006;19:95-100.

-6 Langley RG, Burton E, Walsh N, et al: In vivo confocal scanning laser microscopy of benign lentigines: comparison to conventional histology and in vivo characteristics of lentigo maligna. J Am Acad Dermatol 2006;55:88-97. 